\title{
Finding pathways to synergistic development of Sustainable Development Goals in China
}

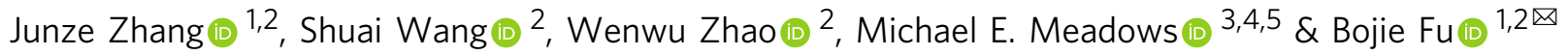

While the Sustainable Development Goal (SDG) index is a widely employed method of measuring progress in the United Nations (UN) SDGs, as it allows comparisons across countries and regions, it does not usually offer any indication as to how to move the SDGs forward. To address this deficit, we analyzed the impact of the offsetting effects of changes in individual SDG scores on the SDG index from 2015 to 2018 at the provincial level in China. Future priority goals are also revealed by accessing the interactions among the SDGs. Our findings show that both the number of SDGs that experienced an increase or decrease in the score and the degree of change in SDG scores have a major impact on changes in the SDG index score. For example, a decline in individual SDG scores at the national level usually represents a decline in the scores of more than 15 provinces, while an increase in the SDG score may mask the fact that some provinces have experienced a decline in their scores. In addition, changes in SDG scores can show some similarities in the strengths and weaknesses even across provinces in different geographical zones. The analysis of SDG interactions suggests that prioritizing the promotion of SDG7 (Affordable and Clean Energy) and SDG12 (Responsible Production and Consumption) will advance the synergistic development of SDGs in China. Overall, our study highlights the necessity to focus not only on regions with declining aggregated index scores but also on goals with declining scores in order to implement the SDGs as a whole. Meanwhile, leveraging the similarity of SDG changes to inform sustainable management policies will contribute to the overall implementation of the SDGs in different regions.

\footnotetext{
${ }^{1}$ State Key Laboratory of Urban and Regional Ecology, Research Center for Eco-Environmental Sciences, Chinese Academy of Sciences, Beijing, China. ${ }^{2}$ State Key Laboratory of Earth Surface Processes and Resource Ecology, Faculty of Geographical Science, Beijing Normal University, Beijing, China. ${ }^{3}$ Department of Environmental \& Geographical Science, University of Cape Town, Rondebosch, Cape Town, South Africa. ${ }^{4}$ School of Geographical and Oceanographic Sciences, Nanjing University, Nanjing, China. ${ }^{5}$ College of Geography and Environmental Sciences, Zhejiang Normal University, Jinhua, China.

凶email: bfu@rcees.ac.cn
} 


\section{Introduction}

ow to effectively monitor and assess the level of sustainable development determines whether people can achieve this vision (Holden et al., 2014; Barbier, 2016). Hundreds of sustainable development indicator frameworks have been developed by various organizations, enterprises, or research institutes (Kates et al., 2005; Kraak et al., 2018). However, as the ambiguity of sustainable development, some indicator frameworks not only differ from each other but even conflict with each other (Mair et al., 2017). The proposal of the United Nations (UN) sustainable development goals (SDGs) and its global indicator framework has provided an important reference for forming a common standard (United Nations, 2015, 2017), but the indicators at the global level may not be applicable at the sub-national level. It makes it necessary for countries to develop appropriate indicators based on the global indicator framework (Salvia et al., 2019; Allen et al., 2020). Hence, monitoring and assessing the progress of SDGs at the sub-national level is generally recognized as one of the priorities in advancing sustainable development (Allen et al., 2021), which is often influenced by the capacity of data monitoring, access, and integration, as well as whether robust assessment methods are available.

There are currently three main forms of measuring progress on SDGs. First, the UN publishes an annual report on SDGs (United Nations, 2021), but this is primarily for progress on SDGs at the global level. Second, most countries agree to monitor changes in SDGs in individual countries by submitting voluntary national review reports to the UN High-Level Political Forum on Sustainable Development (Allen et al., 2018). However, these official government-led reviews tend to be narrative, focusing on the process of policy formulation and discussion of future challenges. While such narrative progress reports are an important way to increase the transparency of voluntary national reviews and to facilitate the sharing of country experiences, the lack of quantitative assessments of specific targets and indicators for each SDG does not provide precise guidance on how to prioritize future tasks and allocate resources (Allen et al., 2018). Hence, the third, quantitative assessment of SDGs has received more widespread attention.

The SDG index, jointly developed by the BertelsmannStiftung and the UN Sustainable Development Solutions Network Group, has become an important tool for measuring the progress of SDGs across countries (Sachs et al., 2016; Schmidt-Traub et al., 2017). This assessment framework has also been applied to sub-national level assessments (Lynch et al., 2019). Nonetheless, similar to other aggregated indices, while the SDG index can measure the progress of SDGs and allow comparisons across regions, it cannot identify where improvements need to be made (Schmidt-Traub et al., 2017). This is because an aggregated index is susceptible to the offsetting effect of changes in its internal indicators, which means that an increase in index scores may be only due to improvements in some indicators masking the deterioration in others (Sachs et al., 2016). Particularly, different regions may face different advantages and challenges, leading to differences in the offsetting effects of changes in SDGs (Gusmão Caiado et al., 2018; Salvia et al., 2019). However, there are still no studies that report in detail the offsetting effects of implementing SDGs and their spatial differences. In addition, considering the interactions among different SDGs, how to overcome the offsetting effects is an additional knowledge gap that needs to be filled.

To address these deficiencies, we conducted an assessment of the offsetting effects of SDGs progress at the provincial level in China (including 31 provinces, excluding Hong Kong, Macau, and Taiwan) and explored priority goals that would facilitate the overall implementation of SDGs. As the most populous country in the world, China's realization of SDGs has huge implications for progress towards sustainability globally and is often, therefore, the object of scrutiny (Lv et al., 2019; Xu et al., 2020). Currently, the Chinese government has taken a series of actions to incorporate SDGs into the development plans of different sectors and has created demonstration zones to explore effective ways to implement SDGs (Wei et al., 2018; Wang et al., 2020). Nonetheless, although some researchers have analyzed the spatial and temporal variations of China's SDGs from 2000 to 2015 (Xu et al., 2020), and the uneven development characteristics identified and brought into focus (Liu et al., 2021), the offsetting effects arising from the changes in SDGs and SDG targets have never been elucidated in detail. Clarifying the offsetting effects on the aggregated index and the solutions to address them offers new insights into measuring the progress towards sustainable development at the regional, national, and global levels.

In this study, we addressed the following questions. First, what are the changing characteristics of SDGs and their offsetting effects in China since SDGs were proposed? Second, what are the spatial similarities and differences in SDG changes among 31 provinces in China? Third, which SDGs should be prioritized for future development to compensate for the offsetting effects among different SDGs? It should be emphasized that we focus on the progress of SDGs after 2015. In so doing, the overall purpose is not on quantifying the temporal variations of SDGs in China, which have already been assessed, but rather to reveal the offsetting effects of changes in an aggregated index and to suggest how these can be minimized or reversed. Therefore, considering the changing characteristics since SDGs were proposed as our study object not only allows us to analyze theoretical solutions as to how to deal with offsetting effects but also ensures the timeliness of results, which may then provide suitable guidelines for future sustainability policy formulation.

\section{Methods}

Indicator selection and data sources. To answer the above research questions and ensure the reliability of the assessment results, we constructed the framework of SDG indicators for China at the provincial level by referring to the global indicator framework (United Nations, 2017) and indicators in the published literature (Sachs et al., 2019; Xu et al., 2020), as well as combining the five principles of policy relevance, universal applicability, reasonable indicativeness, timeliness and statistical reliability (Supplementary Methods). Overall, our indicator framework has a total of 88 indicators, which correspond to 71 targets and 16 goals (Table S1). It should be noted that SDG14 (Life below Water) is mainly related to the marine ecosystem and, while China has 11 provinces adjacent to the sea (not including Hong Kong, Macao, and Taiwan), scores for SDG14 are irrelevant in the 20 inland provinces. Changes in SDG14 are therefore not comparable across all provinces and this goal was excluded from the analysis.

To ensure the statistical reliability, general applicability, and currency of the data, we rely mainly on official statistics from different sectors in China to obtain the data. Although some researchers have recently developed methods of data mining relevant to SDG indicators, such as spatial big data obtained through remote sensing (Estoque, 2020), data produced by such methods typically represent the status of indicators at a particular point in time and are not yet suitable to reveal changes over time. Therefore, such data sources were not considered in our assessment. In total, 19 official statistics, including the China Statistical Yearbook, the China Environmental Statistics Yearbook, and the China Health Statistics Yearbook, were utilized in this study (Table S1). 
Although we are concerned with offsetting effects and spatial differences in changes in SDGs across provinces from 2015 onwards, to obtain reasonable target and baseline values for each indicator, we collected historical data for each indicator from 1990 , or since statistics became available, to 2018 , the years when data could be obtained for all indicators during the data collection period. As can be seen from Fig. S1, the number of indicators for most of SDG targets in this paper does not reach $50 \%$ of the number of global indicator frameworks, but this reflects the fact that it is more difficult to obtain data related to SDG indicators at the sub-national level. Meanwhile, it also shows that our indicator framework remains an imperfect assessment standard and there is a necessity to continuously refine it for assessing SDGs at the sub-national level as data availability improves.

Data processing. We quantify the change of SDGs from 2015 to 2018 in China by improving the methodology for calculating the SDG index (Sachs et al., 2016). Initially, we normalized the raw data for each indicator to a score in the range of 0-100. Subsequently, indicator scores were aggregated by arithmetic means into target scores, which were further aggregated into SDG scores. The SDG index score is the arithmetic mean of different SDG scores. We describe how SDG index scores were calculated detailly in Supplementary information, a process that consists of four parts: selection of indicators, setting of target and baseline values (Table S2), normalization, and aggregation. We analyzed the offsetting effect of changes in SDG index scores by counting how many SDG scores in China and its provinces had increased or decreased and considered the number of targets within each SDG that had increasing or decreasing scores.

In addition, the one-way analysis of variance (ANOVA) was used to test for levels of statistical significance in SDG index scores in each province in 2018. The least significant difference (LSD) method was then used for posthoc pairwise comparisons of the scores (in R package 'agricolae') (Kniss and Streibig, 2018). The Mann-Whitney $U$ test (in R package 'tableone') (Yoshida, 2020) was then used to analyze the statistical significance of any differences in scores between 2015 and 2018. All analyses were performed in R v3.6.1 software (https://www.r-project.org/).

Analysis of spatial variation. To explore the similarities and differences of the 2015-2018 progress towards the SDGs for all 31 provinces, cluster analysis was applied to the score differences. The hierarchical clustering algorithm assumes that each sample point is a separate cluster class, and then merges clusters with higher similarity in each successive iteration of the algorithm (Nielsen, 2016). This process was repeated until the preset number of clusters was reached, or only a single cluster remained. We first calculated the Euclidean distance between the data set samples (using the $\operatorname{dist}($ ) function in R), and then obtained the hierarchical structure of 31 provinces through the ward sum of square deviation method (using the hclust() function in R). We determined the appropriate number of clusters by calculating gap statistics (Tibshirani et al., 2001), obtained through clusGap() in the "cluster" package in R. The gap statistic was proposed by Tibshirani et al. (2001) to compare the total within intra-cluster variation for different values of $k$ with their expected values under null reference distribution of the data. The number of clusters can be chosen as the smallest value of $k$ such that the gap statistic is within one standard deviation of the gap at $k+1$. This means that the clustering structure is far away from the random uniform distribution of points (Tibshirani et al., 2001). Our results show that the 31 provinces can be clustered into four groups (Fig. S2A). The final clustering results are shown in Fig. S2B.
Interaction analysis. To explore which SDGs could be prioritized to mitigate the offsetting effects of changes in SDGs across provinces, we analyzed the interactions (synergies and trade-offs) between different SDGs and their changing characteristics between 2015 and 2018. To ensure the practical implications of the results, we assessed interactions among SDGs based on the systematic classification framework of SDGs (Fu et al., 2019), which divided the 16 SDGs analyzed in this paper into three categories, namely "Essential Needs", "Governance" and "Objectives". For a detailed explanation of the SDGs systematic classification framework, please see Fig. S3. Multiple factor analysis (MFA) was used to quantify the interactions among different SDG categories and identify the SDGs that have the strongest influence within each category by significance analysis. MFA is a form of correlation analysis, and not a hypothesis test of causality, but can analyze three or more groups of variables (Abdi et al., 2013). The correlation between different variable categories is measured by the RV coefficient, which is a measure of the similarity between square symmetric matrices (Josse et al., 2008). In statistics, the RV coefficient is a multivariate generalization of the squared Pearson correlation coefficient (the range is between 0 and 1), which can be tested by the permutation method (Josse et al., 2008; Ding et al., 2016). Here, we calculated RV coefficients for the three SDG categories in 2015 and 2018 using crosssectional data of SDG scores by provinces, respectively.

The MFA in the study was conducted using the function $\mathrm{mfa}()$ of the "FactoMineR" package in R (Borcard et al., 2018). Although the RV coefficients themselves do not reflect the directionality of

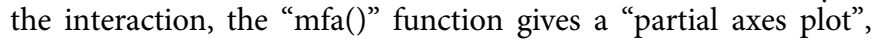
which reflects the projection of the principal components of different data groups onto the global PCA (Borcard et al., 2018). The angle between the principal components can indicate the direction of interaction, where an acute angle represents positive correlation (synergy), an obtuse angle is a negative correlation (trade-off), and an approximately orthogonal angle means no correlation. We, therefore, determined the overall interaction characteristics (synergy or trade-off) of three SDG categories based on the angle between the first principal components in the partial axes plots (Borcard et al., 2018). Additionally, we also calculated Pearson correlation coefficients $\left(r_{\mathrm{p}}\right)$ between the SDG scores in 2015 and 2018 to further elucidate the specific forms of interactions between and within each SDG category. To avoid over-interpretation of correlations, we defined values of $r_{\mathrm{p}}$ located in four different intervals $[-1,-0.5],(-0.5,0],(0,0.5)$, and $[0.5$, $1]$ as the trade-off, weak trade-off, weak synergy, and synergy, respectively, concerning the experience of relative studies (Warchold et al., 2020).

\section{Results}

Progress and offsetting effects. In terms of overall progress towards achieving the SDGs, China's SDG index score was 65.4 in 2018 (Fig. 1A), which is a marginal improvement compared to 2015 ( $P>0.05$, Fig. 1B). However, progress has been uneven across 31 provinces. Our results (Fig. 1A) show that, in 2018, among the 31 provinces, Beijing has the highest ranking (79.3), and Gansu has the lowest (57.7). Eight provinces have SDG index scores higher than the national mean value (65.4), while scores in 22 provinces are lower than the national level (Fig. 1A). Nevertheless, results of the ANOVA show that differences in scores between 2015 and 2018 are statistically significant $(P<0.05)$ in only a few provinces (Fig. 1A). Individually, Climate Action (SDG13) is China's best-performing SDG in 2018, while Life on Land (SDG15) has progressed least (Fig. S4). Most provinces exhibit broadly similar trends, although there are some notable outliers. Ningxia, for example, has performed especially poorly in 
A

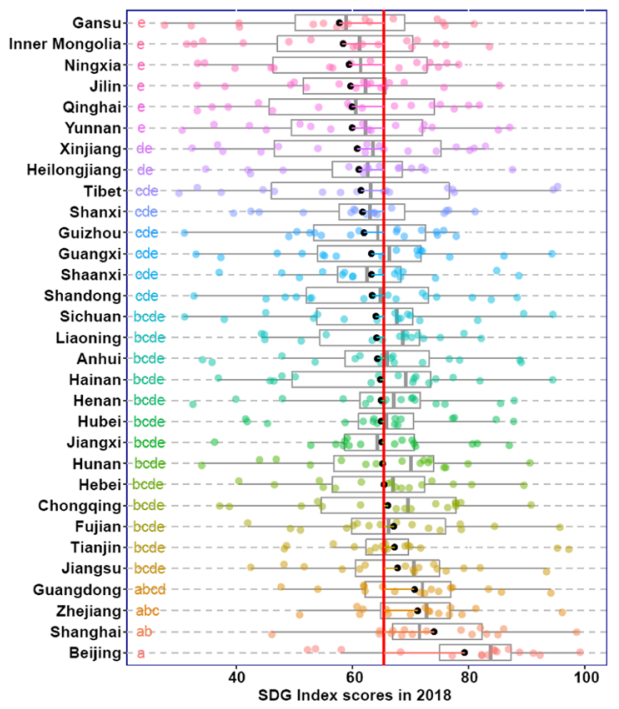

B Innerm

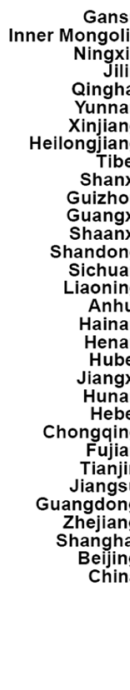

Mingx
Jilin-

Yunnai-

Xinjiang

ngjiang-

Tibet-
Shanxi-

Shanxi-

Shangxi-

Shandong

Sichuan-

Liaoning-
Anhui-
Hainan-

Henan

Hubei-

Jiangxi-

Hebei-

Fujian-

Fujian
Tianjin-

Jiangsu-

nejiang-

Beijing-
China-

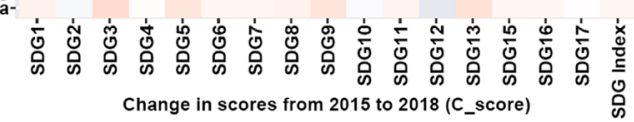

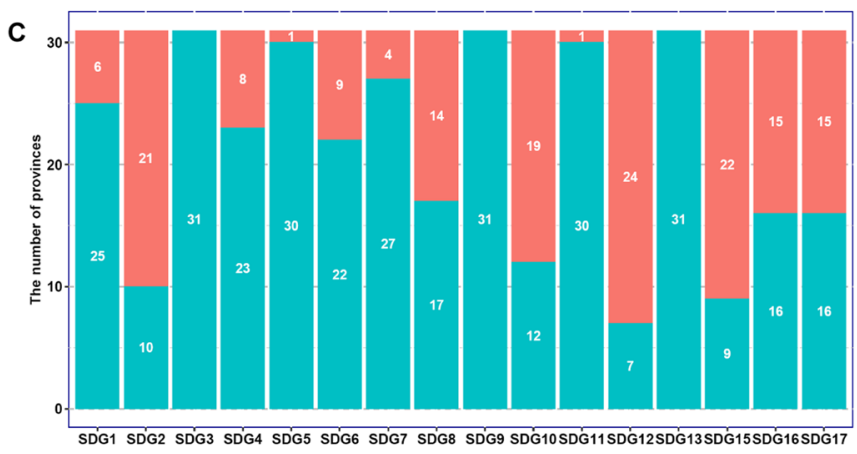

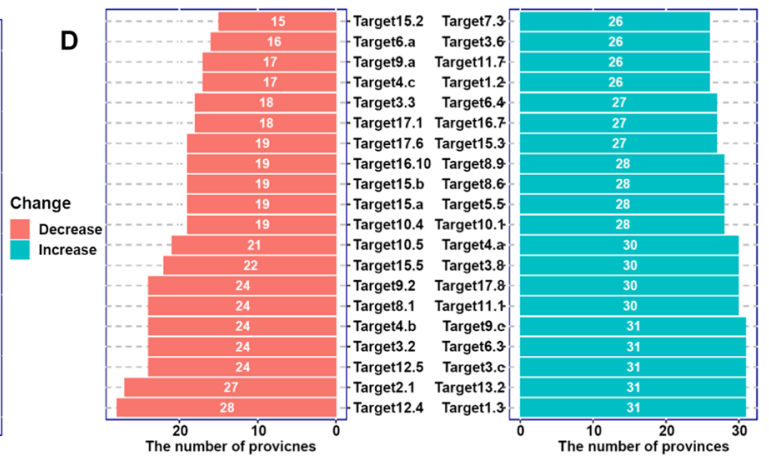

Fig. 1 SDG index and individual SDG scores (2018) and their changes (2015-2018). A SDG index scores for China (red line) and 31 provinces in 2018. The different lowercase letters in the graph represent significant differences $(P<0.05)$ in scores among 31 provinces. B Changes in SDG index scores for China and 31 provinces between 2015 and 2018. C The number of provinces in which each SDG score increased or decreased between 2015 and 2018. D The number of provinces in which each SDG Target score (top 20) increased or decreased between 2015 and 2018.

terms of responsible consumption and production (SDG12) (Fig. S4).

Regarding offsetting effects, the results indicate that a declining SDG score at the national level is exhibited when the score is lower across at least 15 provinces. On the other hand, a national level increase in a particular SDG score may conceal the fact that there is a reduction in the scores in most provinces. For example, Fig. 1B shows that scores for SDG2 (Zero Hunger), SDG10 (Reduced Inequalities), and SDG12 (Responsible Production and Consumption) decreased between 2015 and 2018 and that scores for these SDGs decreased in more than 15 provinces (Fig. 1C), although these changes are statistically not significant $(P>0.05$, see Table S3). However, although the scores for SDG15 (Life on Land), SDG16 (Peace, Justice and Strong Institutions), and SDG17 (Partnerships for the Goals) all increased at the national level, the scores for these three SDGs decreased in more than 15 provinces.

In addition, the offsetting effect was also observed in the Targets within each SDGs. For example, although SDG3 (Good Health and Well-Being) has increased in all provinces (Fig. 1C), the scores for Target3.2 (Reducing Child Mortality) and Target3.3 (Eliminating Infectious Diseases) declined (in 24 and 18 provinces, respectively) (Fig. 1D). The increase in SDG3 scores occurred mainly due to improvements in Targets3.c (Increase Health Worker Density), 3.8 (Improve Coverage of Basic Health Services), and 3.6 (Reduce Traffic Fatalities) (Fig. 1D). In the same period, the score for SDG9 (Industry, Innovation, and Infrastructure) increased in all provinces, but the scores of
Target9.2 (Industrial Value Added as a Percentage of GDP) and Target9.a (Investment in Environmental Pollution Control as a Percentage of GDP) have decreased in 24 and 17 provinces, respectively. The improvement observed in mobile network coverage (Target9.c) is the main reason for the increase in the SDG9 scores across all provinces.

Spatial variations in the progress of SDGs. From the change in SDG scores by province, the degree of change in individual SDG scores strongly impacts changed in the SDG index scores. It is found that, although the number of SDGs that experienced declining scores in Jiangsu, Shandong, and Shanghai was smaller than the number of SDGs that experienced an increase (Fig. 2A), the SDG index scores in all three regions exhibited a decline between 2015 and 2018 (Fig. 1B). This is due largely to the disproportionate reductions in particular SDG scores in these three provinces, which effectively negate improvements in the other SDG scores. For example, Jiangsu's SDG16 dropped by 17 points; Shandong's SDG12 dropped by 18.7 points; and Shanghai's SDG2 dropped by 25.7 points (Fig. 1B). Meanwhile, although the number of SDGs with decreasing scores in Jilin is slightly higher than the number of SDGs with increasing scores (Fig. 2A), the decrease in scores for other SDGs is offset by an average increase of more than 8 points for SDG3, SDG5, and SDG13 (Fig. 2B).

In addition, Fig. 2A shows that even though the 2018 SDG index scores are higher in the east. Western provinces exhibit a greater number of SDGs with declining scores. For example, among the provinces in east China, the number of SDGs with 

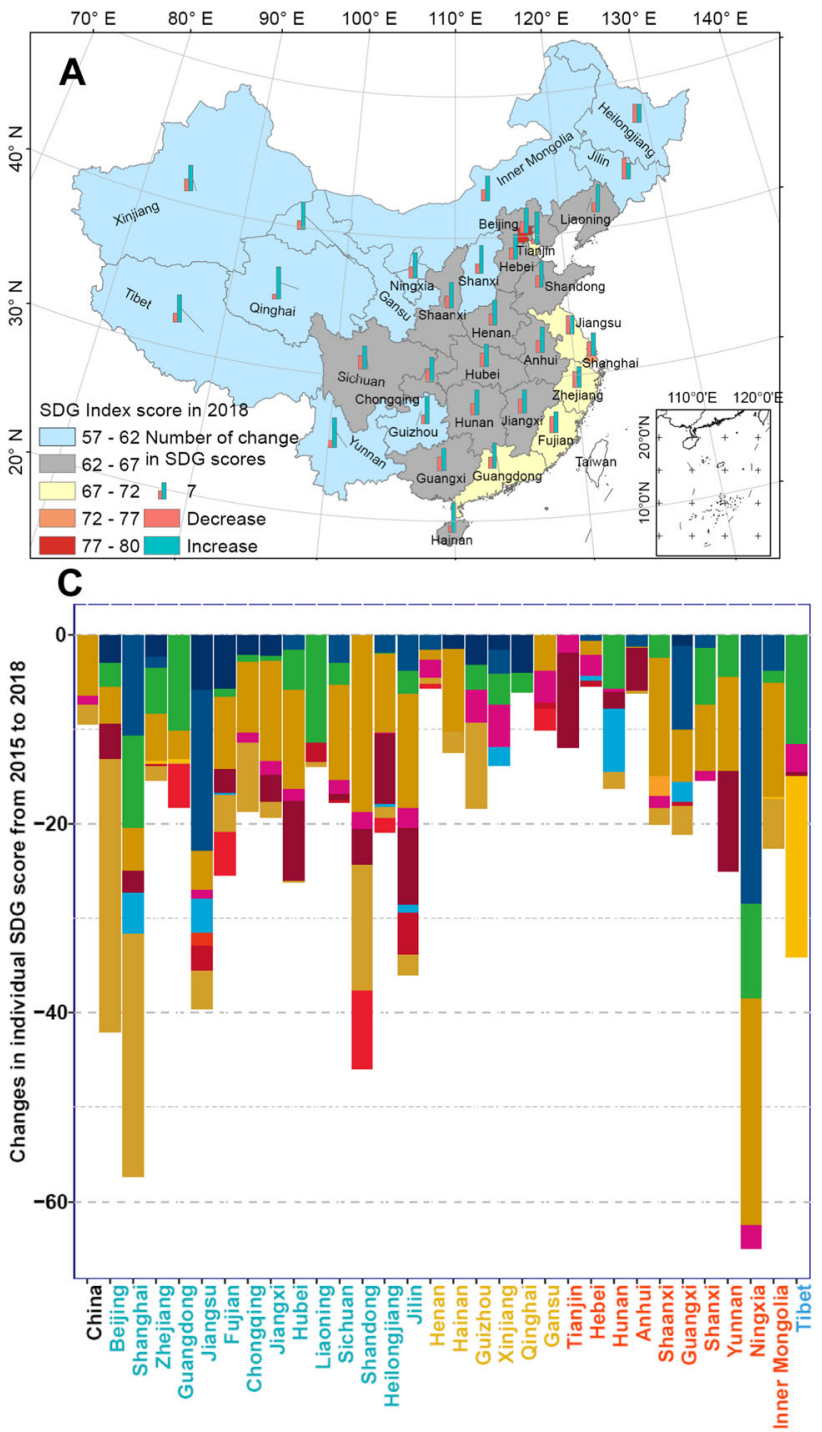

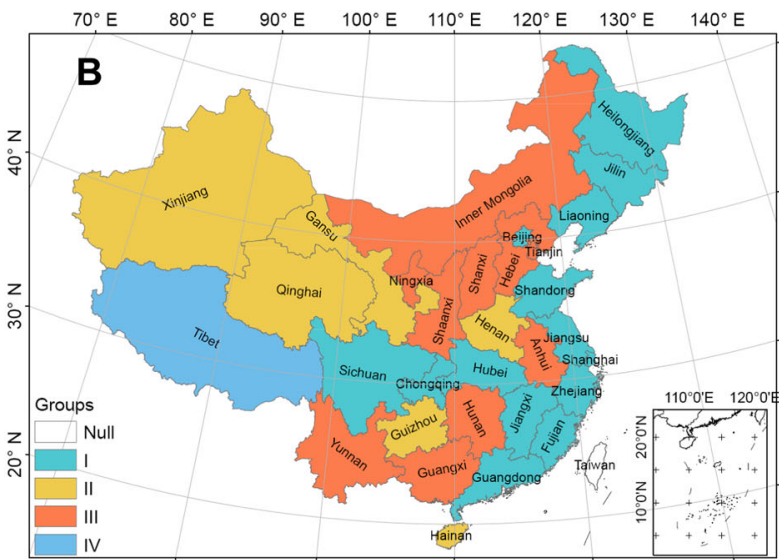

D

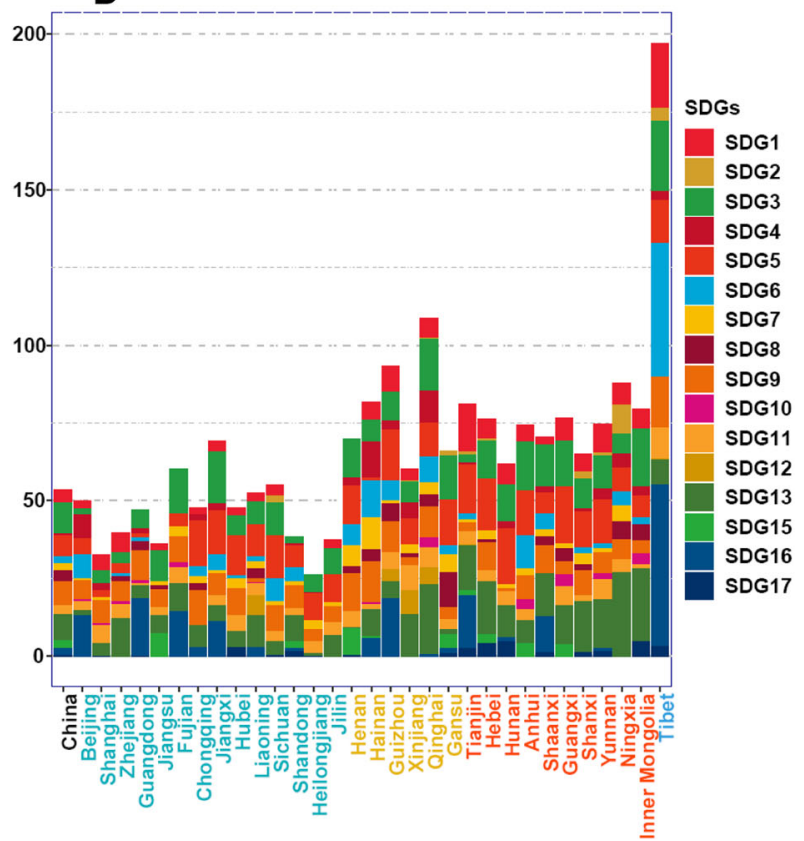

Fig. 2 Spatial characteristics of the SDG scores and their changes from 2015 to 2018 in China. A Spatial distribution of SDG index scores for 31 provinces in 2018 (polygons with different colors); light green (red) bars indicate numbers of SDGs that increased (decreased) from 2015 to 2018 . B Results of cluster analysis of SDG score changes for 31 provinces from 2015 to 2018. C SDG scores that declined from 2015 to 2018 were classified into the four groups of provinces identified in the cluster analysis. D SDG scores that increased from 2015 to 2018 were classified into the four groups of provinces identified in the cluster analysis.

decreasing scores generally exceeds five, and as many as seven and eight in Zhejiang and Jiangsu provinces, respectively. However, among the provinces in northwest China, the number of SDGs with decreasing scores generally is below five, and as few as two SDGs in Qinghai (SDG15 and SDG17). More specifically, although provinces in northwest, southwest, and northern China had low overall SDG index scores (57-62) in 2018, these provinces have more SDGs with increasing scores, suggesting higher potential for sustainable development. On the other hand, the central, southern, eastern, and northeastern provinces, despite having relatively high SDG index scores (62-72), exhibit a greater number of SDGs with declining scores and therefore may face constrained growth. Heilongjiang and Jilin provinces in northeast China face the most serious development challenges in not only exhibiting low overall SDG index scores (61.1 and 59.7, respectively) but also declining scores in more than eight SDGs.

Using hierarchical clustering, we further analyzed the similarities and differences in the patterns of variation in SDG scores across provinces from 2015-2018. The appropriate number of clusters is calculated using the gap statistic (Tibshirani et al.,
2001), revealing that the 31 provinces can be clustered into four groups (Fig. 2B; and see Fig. S2 for detailed information). Similarities and differences in the changes in the SDG scores of the four groups of provinces can be summarized in the patterns of increasing and decreasing SDG scores, respectively.

For SDGs with declining scores (Fig. 2C), provinces in groups I, II, and III generally show a downward trend in scores for SDG12, SDG15, and SDG16. However, among Category I provinces, a more marked decline in scores for SDG2 and SDG8 (Decent Work and Economic Growth) is evident. For provinces in group II, the decline in scores for SDG10 is more pronounced, while those in group III appear to be less affected by the decline in SDG2, SDG8, and SDG10, and more by the decline in SDG12, and in some of these provinces the decline in SDG6 (Clean Water and Sanitation) and SDG16 is greater. Finally, in group IV, comprising only the Tibet Autonomous Region, the most obvious difference is the very marked decline in the score for SDG7 (Affordable and Clean Energy) (Fig. 2C).

From the perspective of rising scores (Fig. 2D), the scores for SDG1 (No Poverty), SDG3, SDG4 (Quality Education), SDG5 

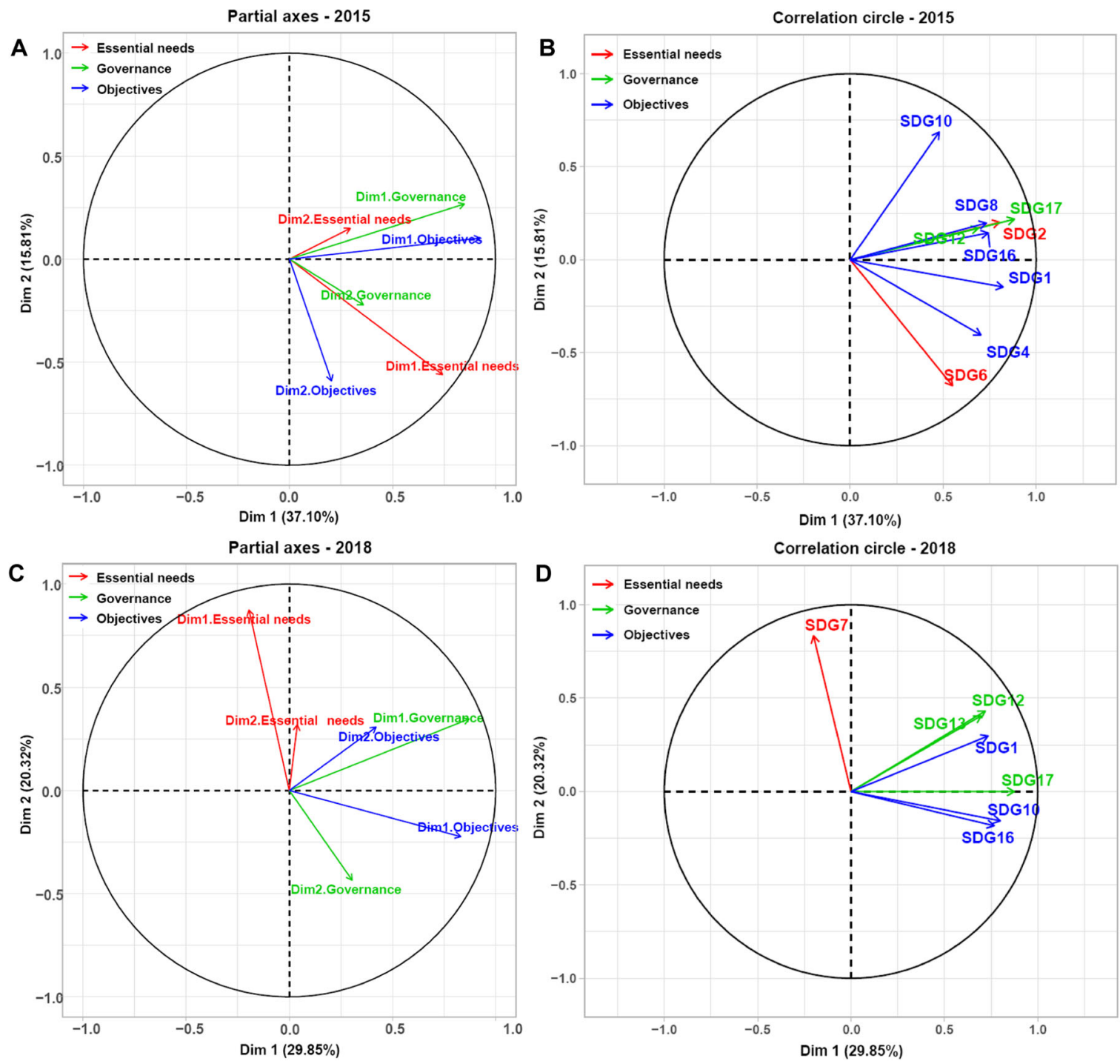

Fig. 3 Interactions among different SDG categories and the goals that significantly affect different interactions in China. A Partial axes: Interactions among different SDG categories in 2015. B Correlation circle: Goals that significantly influence interactions among different SDG categories in 2015. C Partial axes: Interactions among different SDG categories in 2018. D Correlation circle: Goals that can significantly influence interactions among different SDG categories in 2018. The partial axes reflect the principal components of different data groups in the global principal component analysis, which indicate general characteristics of interaction across the three SDG categories. Correlation circles reveal the SDGs that have a significant effect in each category. Among the three SDG categories, "Essential Needs" consists of SDG2 (Zero Hunger), SDG6 (Clean Water and Sanitation), SDG7 (Affordable and Clean Energy), and SDG15 (Life on Land); "Objectives" includes SDG1 (No Poverty), SDG3 (Health and Well-Being), SDG4 (Quality Education), SDG5 (Gender Equality), SDG8 (Decent Job and Economic Growth), SDG10 (Reduced Inequalities) and SDG16 (Peace, Justice and Strong Institutions);

"Governance" includes SDG9 (Industry, Innovation, and Infrastructure), SDG11 (Sustainable Cities and Communities), SDG12 (Responsible Production and Consumption), SDG13 (Climate Action) and SDG17 (Partnerships for the Goals). Please note that SDG14 (Life below Water) is not considered in this assessment.

(Gender Equality), SDG9 (Industry, Innovation, and Infrastructure), and SDG11 (Sustainable Cities and Communities) generally improved, but there were obvious differences in the extent to which the SDG scores increased in different provinces. Group I provinces exhibited very little improvement in SDG scores, with somewhat greater increases in provinces of groups III and II, more especially in SDG10 in group III. The most prominent increases in SDG scores occurred in the group IV province, especially in SDG6 and SDG16 (Fig. 2D).

Synergies and trade-offs among SDGs. Our results show that statistically significant positive correlations, and therefore synergies, are evident among the three SDG categories in 2015
(Fig. 3A). Specifically, the RV coefficients are 0.402 (between "Essential Needs" and "Objectives"), 0.307 (between "Governance" and "Essential Needs"), and 0.538 (between "Governance" and "Objectives"), respectively. Moreover, there are no statistically significant trade-offs $\left(-1 \leq r_{\mathrm{p}} \leq-0.5\right)$ for all SDGs within or between each category and only a few weak trade-offs $\left(-0.5<r_{\mathrm{p}} \leq 0\right)$ (Fig. S5A). For example, interactions between "Essential Needs" and "Objectives" exhibit eight weak trade-offs, but also six statistically significant synergies $\left(0.5 \leq r_{\mathrm{p}} \leq 1\right)$ and 14 weak synergies $\left(0<r_{\mathrm{p}}<0.5\right)$ (Fig. S5B). Weak trade-offs occur mainly between SDG7 and the SDGs in "Objectives", while synergies are stronger among SDG2, SDG6, and the SDGs in "Objectives". Figure 3B indicates those SDGs that have made a significant contribution to interactions between the SDG 
categories. For example, SDG2 and SDG6 play a major role in "Essential Needs", this can also explain the positive interactions between "Essential Needs" and other categories. In addition, SDG12 and SDG17 play a major role in "Governance" and, except for SDG3, all SDGs make a significant contribution to "Objectives".

Considering the situation in 2018, however, reveals that synergies between various SDGs have weakened (Fig. 3C). Although there is still a significant positive correlation between "Governance" and "Objectives" (RV $=0.396, \quad P<0.01)$, the correlations between "Governance" and "Essential Needs" have weakened ( $\mathrm{RV}=0.109, P>0.05)$. Moreover, SDGs representing "Essential Needs" and "Objectives" exhibit no correlation $(\mathrm{RV}=0.098, P>0.05)$. From Fig. S6, we can see that in 2018 there was no longer a synergy between the SDGs within "Essential Needs" and "Objectives", mainly because SDG2 and SDG6 within the "Essential Needs" no longer have a synergy with all the SDGs within the "Objectives". Meanwhile, there was also an increase in the number of weak trade-offs among SDG7, SDG15, and the SDGs in the "Objectives" (Fig. S6). Figure 3D indicates that SDG7 makes a significant contribution to "Essential Needs", which explains the weakening of synergies among "Essential Needs" and other categories. Therefore, SDG7 should be considered as a priority goal across all provinces to promote balanced development in China. Meanwhile, as SDG12 plays a significant role in "Governance" and SDG12 scores in each province have decreased to varying degrees, accelerating the implementation of SDG12 in the future may contribute positively to the achievement of all or many SDGs.

\section{Discussion}

The offsetting effects on advancing the SDGs. The key to advancing the SDGs as a whole is to avoid offsetting progress in some SDGs through the deterioration in others (Sachs et al., 2016), while attention is also required to see if there have been declining scores in some regions that are masked by progress in others (Salvia et al., 2019). Past assessments have mainly discussed the offsetting effects of implementing the SDGs qualitatively (United Nations, 2021) but quantification of results should help to provide more reliable information on which to base sustainable development policies. Our findings show that the number of increases or decreases in SDG indicators and the degree of change in individual SDG scores impact the SDG index scores. This implies that progress in a country's SDG index score may be based on an improvement only in some goals, thus outweighing the decline in others, or that improvements in some regions mask the deterioration in others.

Previous assessments have indicated that the improvement in China's level of sustainable development has benefited mainly from positive developments in social and economic related SDGs, such as poverty reduction (SDG1) and urbanization (SDG11), as well as in promoting educational (SDG4) and gender (SDG5) equity (Lv et al., 2019; Xu et al., 2020). Nevertheless, our study highlights that the decline in SDG2 and SDG12 scores are widespread across the country. Despite consistently increasing food production in China, a reduction in food safety has occurred, although it has not received sufficient attention (Afshin et al., 2019). It is possible that food safety issues are related to irrational consumption patterns, which have led to SDG2 and SDG12 showing a simultaneous decline.

Additionally, while the uneven implementation of SDGs among provinces has been identified (Liu et al., 2021), our results indicate that provinces with quite different geographical circumstances and contrasting economic levels may face similar development dilemmas. For example, China's eastern coastal and central regions generally have a much higher level of socioeconomic development compared to northeastern China (Lv et al., 2019), but they could be classified in the same cluster and exhibit declining scores for SDG2, SDG8, and SDG12. However, the classification of Tibet into a separate group is noteworthy, especially as most studies of development have tended to pair Qinghai and Tibet as a single entity (the Qinghai-Tibet Plateau) (Cao and Zhang, 2015). In this case, while both Tibet and Qinghai face similar challenges in biodiversity conservation (SDG15), the two provinces differ markedly in other challenges. For example, Tibet is falling well short of achieving SDG7 (Affordable and Clean Energy), while Qinghai province has become a model province for clean energy construction in China (Dong et al., 2020). Hence, these results suggest that the traditional local-based zoning may be inappropriate and that the identified clusters may facilitate a more integrated approach to improve policy consistency, and thus contribute to more balanced development across provinces.

Future priorities. Our findings suggest that SDG7 and SDG12 should be prioritized to accelerate the implementation of SDGs in China by 2030 and to promote balanced development across provinces. This is largely because SDG7 and SDG12 play a significant role in the interactions (weak synergies) among "Essential Needs" and "Governance" with other SDG categories, respectively. Considering changes in SDG scores between 2015 and 2018, we found that SDG7 scores have risen inconsistently and are relatively higher in the group II provinces, while SDG12 scores have fallen in most provinces. Based on these results, it will be important to encourage balanced progress in SDG7 across all provinces in China, while also reversing the decline of SDG12 scores.

Clearly, clean energy from wind, water, solar power, and other renewable sources has become an increasingly necessary tool to combat the energy crisis and climate change (Dincer, 2000; Gunnarsdottir et al., 2020). However, while renewable energy can mitigate the effects of energy shortages, it is also limited by natural conditions, such as the availability of adequate hydro, wind, and solar resources (Yillia, 2016). In China, solar and hydropower are mainly located in the northwestern and southwestern provinces (Zhang et al., 2017), while most provinces are still dominated by thermal power generation, which has led to inconsistent progress in SDG7 across provinces. To achieve the commitment of the carbon emission peak before 2030 and carbon neutrality before 2060, the Chinese government needs to increase the primary energy share of renewables in all provinces and ensure the affordability of clean energy for different social groups (Gallagher et al., 2019). However, attention also needs to be paid to the negative environmental and social impacts of clean energy construction, such as the potential consumption of agricultural or forest land by energy facilities, and competition for water between the energy and agricultural sectors (D'Odorico et al., 2018). Therefore, considering the nexus among different sectors will be an important pathway to promote clean energy development in all provinces.

For responsible production and consumption (SDG12), irrational human activities have been shown to be major causes of environmental degradation (Barbier, 2016; Clark and Harley, 2020), and improving human production and consumption patterns will be essential to maintaining critical Earth system processes within planetary boundaries (Shaikh et al., 2021). Nonetheless, there are still major or significant challenges to the progress of SDG12 in most countries, especially in developed and developing countries (Sachs et al., 2019). Some have even suggested that the trade-offs between SDG12 and other SDGs 
may be impossible to overcome (Pradhan et al., 2017; Kroll et al., 2019). In this study, SDG12 was classified into the "Governance" category, which is expected to play a coordinating role to reduce the consumption of "Essential Needs" while promoting the SDGs associated with "Objectives" (Fu et al., 2019). Our results show that the decline in SDG12 scores diminishes the coordinating role of "Governance" and that SDG12 exhibits synergies with other SDGs (Figs. S5 and S6). This provides quantitative evidence for advancing the achievement of other SDGs by accelerating SDG12. It is important to note, however, that it is difficult to find measures with universal applicability to reverse the decline in SDG12 scores since it is affected by multiple factors such as people's reliance on past development patterns or cultural differences (Gusmão Caiado et al., 2018; Shaikh et al., 2021). Nevertheless, it is crucial to guide the transformation of human behaviors through top-down policies, and there is also a need to incentivize bottom-up behaviors to explore transformative approaches that apply to different regions (Scoones et al., 2020).

Implications for the rest of world. While this paper focuses on the offsetting effect of the short-term implementation of SDGs in China and its potential solutions, our results provide some insights for other countries regarding integrated assessments of sustainable development. Some studies have questioned whether the implementation of SDGs represents the achievement of actual sustainable development (Wackernagel et al., 2017; Jain and Jain, 2020). For example, it has been suggested that if sustainable development is characterized by a UN Human Development Index (HDI) value $>0.8$ and an ecological footprint per person $<1.7$ gha, no country can currently meet such a requirement (Wackernagel et al., 2017). Countries with high SDG index scores have been shown to exhibit both high HDI values and a large per capita ecological footprint, implying that currently developed countries may have achieved high socio-economic levels at the cost of environmental degradation and through the depletion of natural resources in other countries (Wackernagel et al., 2017; Jain and Jain, 2020). Our study further highlights the need to focus on SDGs that have declined even when SDG index scores have improved. In other words, while the aggregated index allows people to quickly measure and compare sustainability levels across different regions, to provide precise guidance for improving regional sustainable development policies, a targeted analysis of changes in indicators under different themes is needed to elucidate the offsetting effects that influence the change of an aggregated index.

In addition, shifting away from a siloed development pathway is also necessary for the overall implementation of SDGs (Stafford-Smith et al., 2017; Lim et al., 2018). Our results show that classifying the strengths and weaknesses of various regions may facilitate the achievement of policy coherence. However, the classification of different development dilemmas can only provide a basis for joint management, while effectively promoting balanced development in different regions requires not only the coordination of top-level leaders but also the spontaneous collaboration of grassroots organizations (Fu et al., 2020). Illuminating the synergies and trade-offs among different SDGs may be a powerful way to address the offsetting effects (Nilsson et al., 2018). Although the interactions among different SDGs obtained from correlation analysis do not represent causality (Pradhan et al., 2017), the systematic classification framework of SDGs used in this paper implies that there are causal links among SDGs (Fu et al., 2019). Accordingly, the analytical framework is applicable to other countries or regions to explore future priority goals.
Limitations and perspectives. The indicator-based approach is imperative for monitoring and assessing sustainable development, as it provides a basis on which to judge whether sustainable development is being achieved, to analyze the problems, and to understand their complexity while providing a reference for policymaking (Meadows, 1998; Mair et al., 2017). However, such assessment results are very sensitive to the selection of indicators (Kates et al., 2005). While the indicators used in this paper are informed by the official indicator framework as well as indicators from the literature, there is still no consensus as to what constitutes a suitable SDG indicator framework at the sub-national level and indicators may be challenged or replaced by others in due course. Data availability is an additional constraint in that most of SDGs in our indicator framework exhibit below $50 \%$ of the official number of indicators. These deficiencies contribute to some uncertainty in the current results and thus it will be important in the future to locate and apply more data monitoring tools to better disclose the offsetting effects of SDGs and their influencing factors.

Our analysis of the interactions among different SDGs can offer only pointers to identifying future priorities because different classifications of SDGs may impact the results (Lim et al., 2018). The analytical framework used in this paper is an important perspective on SDG interactions, but current results describe the linear relationships among SDGs. However, non-linear interactions, complex causal feedbacks among different SDGs, and their cross-scale effects all have a profound impact on the overall implementation of SDGs (Kroll et al., 2019; Anderson et al., 2021), although these gaps lie beyond the scope of this paper. Accordingly, as the SDG indicator framework is being refined at different scales, more effort needs to be devoted to monitoring the offsetting effects of SDGs, their interactions, and whether they can be achieved within the constraints of the planetary boundaries. Nevertheless, despite these deficiencies, our findings and analytical framework offer insights for future sustainable development policy development and provide a basis for further research.

\section{Conclusions}

By constructing a framework of SDG indicators applicable to China at the provincial level, we assess the offsetting effects of changes in China's SDGs from 2015 to 2018 and their spatial variations, as well as the priority goals that will facilitate the overall implementation of SDGs. Our findings suggest that offsetting effects have a significant impact on SDG index scores, mainly as a function of the amount of increase or decrease in the scores of different SDGs, and the extent of change in individual SDG scores. Moreover, we also found that provinces in different geographical zones may face the same development dilemma. Effectively exploiting the similarities in the strengths and weaknesses of SDG implementation in different regions can facilitate the achievement of policy coherence, thus promoting balanced development across regions. Additionally, the results of SDG interactions analysis indicated that accelerating the transformation of production and consumption patterns (SDG12), and ensuring energy supply through the promotion of clean energy construction (SDG7) should be prioritized to boost progress towards achieving SDGs in China. Overall, how to achieve the overall implementation of SDGs remains a huge challenge worldwide. Considering that the realization of SDGs is a time-critical task, climate change, disease (not least the COVID-19 pandemic) and fragile international partnerships have also exacerbated uncertainties. Our results highlight the importance for countries to focus their future management policies not only on areas with low (declining) SDG index scores but also on areas with high SDG index scores where several SDGs have underperformed. 


\section{Data availability}

The datasets generated during and/or analyzed during the current study are not publicly available due to confidentiality issues but are available from the corresponding author on reasonable request.

Received: 27 July 2021; Accepted: 6 January 2022;

Published online: 18 January 2022

\section{References}

Abdi H, Williams LJ, Valentin D (2013) Multiple factor analysis: principal component analysis for multitable and multiblock data sets. WIREs Comput Stat 5:149-179

Afshin A, Sur PJ, Ferrara G et al. (2019) Health effects of dietary risks in 195 countries, 1990-2017: a systematic analysis for the Global Burden of Disease Study 2017. Lancet 393:1958-1972

Allen C, Reid M, Thwaites J et al. (2020) Assessing national progress and priorities for the Sustainable Development Goals (SDGs): experience from Australia. Sustain Sci 15:521-538

Allen C, Metternicht G, Wiedmann T (2018) Initial progress in implementing the Sustainable Development Goals (SDGs): a review of evidence from countries. Sustain Sci 13:1453-1467

Allen C, Metternicht G, Wiedmann T (2021) Priorities for science to support national implementation of the sustainable development goals: a review of progress and gaps. Sustain Dev 29:635-652

Anderson CC, Denich M, Warchold A et al (2021) A systems model of SDG target influence on the 2030 Agenda for Sustainable Development. Sustain Sci. https://doi.org/10.1007/s11625-021-01040-8

Barbier EB (2016) Sustainability and development. Annu Rev Resour Econ $8: 261-280$

Borcard D, Gillet F, Legendre P (2018) Numerical ecology with R, 2nd edn. Springer

Cao SX, Zhang JZ (2015) Political risks arising from the impacts of large-scale afforestation on water resources of the Tibetan Plateau. Gondwana Res 28:898-903

Clark WC, Harley AG (2020) Sustainability science: toward a synthesis. Annu Rev Env Resour 45:1-56

Dincer I (2000) Renewable energy and sustainable development: a crucial review. Renew Sust Energ Rev 4:157-175

Ding Y, Liu G, Zang R et al. (2016) Distribution of vascular epiphytes along a tropical elevational gradient: disentangling abiotic and biotic determinants. Sci Rep 6:19706

D'Odorico P, Davis KF, Rosa L et al. (2018) The global food-energy-water nexus. Rev Geophys 56:456-531

Dong L, Li Y, Liu F et al. (2020) Development path and practice of regional fully clean power supply: a case study of Qinghai Province. J Global Energy Intercon 3:385-392. (in Chinese)

Estoque RC (2020) A review of the sustainability concept and the state of SDG monitoring using remote sensing. Remote Sens-Basel 12:1770

Fu BJ, Wang S, Zhang JZ et al. (2019) Unravelling the complexity in achieving the 17 sustainable development goals. Natl Sci Rev 6:386-388

Fu BJ, Zhang JZ, Wang S et al. (2020) Classification-coordination-collaboration: a systems approach for advancing Sustainable Development Goals. Natl Sci Rev $7: 838-840$

Gallagher KS, Zhang F, Orvis R et al. (2019) Assessing the Policy gaps for achieving China's climate targets in the Paris Agreement. Nat Commun 10:1256. https://doi.org/10.1038/s41467-019-09159-0

Gunnarsdottir I, Davidsdottir B, Worrell E et al. (2020) Review of indicators for sustainable energy development. Renew Sust Energ Rev 133:110294

Gusmão Caiado RG, Leal Filho W, Quelhas OLG et al. (2018) A literature-based review on potentials and constraints in the implementation of the sustainable development goals. J Clean Prod 198:1276-1288

Holden E, Linnerud K, Banister D (2014) Sustainable development: Our Common Future revisited. Global Environ Chang 26:130-139

Jain P, Jain P (2020) Are the Sustainable Development Goals really sustainable? A policy perspective. Sustain Dev 28(6):1642-1651

Josse J, Pagès J, Husson F (2008) Testing the significance of the RV coefficient. Comput Stat Data Anal 53:82-91

Kates RW, Parris TM, Leiserowitz AA (2005) What is Sustainable Development? Goals, indicators, values, and practice. Environment 47:8-21

Kniss A, Streibig J (2018) Statistical analysis of agricultural experiments using R. 5 analysis of variance (ANOVA). https://rstats4ag.org/anova.html. Accessed 27 July 2021
Kraak M, Ricker B, Engelhardt Y (2018) Challenges of mapping sustainable development goals indicators data. ISPRS Int J Geo-Inf 7:482. https://doi.org/ 10.3390/ijgi7120482

Kroll C, Warchold A, Pradhan P (2019) Sustainable Development Goals (SDGs): are we successful in turning trade-offs into synergies. Palgrave Commun 5. https://doi.org/10.1057/s41599-019-0335-5

Lim MML, Søgaard Jørgensen P, Wyborn CA (2018) Reframing the sustainable development goals to achieve sustainable development in the Anthropocene-a systems approach. Ecol Soc 23(3):22. https://doi.org/10.5751/ES-10182-230322

Liu YL, Du JQ, Wang YF et al. (2021) Evenness is important in assessing progress towards sustainable development goals. Natl Sci Rev 8:a238. https://doi.org/ 10.1093/nsr/nwaa238

Lv YL, Zhang YQ, Cao XH et al. (2019) Forty years of reform and opening up: China's progress toward a sustainable path. Sci Adv 5:u9413

Lynch A, LoPresti A, Fox C (2019) The 2019 US Cities Sustainable Development Report. Sustainable Development Solutions Network (SDSN), New York

Mair S, Jones A, Ward J et al. (2017) A critical review of the role of indicators in implementing the Sustainable Development Goals. In: Leal Filho W (eds) Handbook of sustainability science and research. World sustainability series. Springer, Cham

Meadows D (1998) Indicators and information systems for sustainable development. The Sustainability Institute. https://edisciplinas.usp.br/pluginfile.php/ 106023/mod_resource/content/2/texto_6.pdf. Accessed 16 Nov 2021

Nielsen F (2016) Hierarchical clustering. In: Mackie I (ed.) Introduction to HPC with MPI for data science Undergraduate topics incomputer science. Undergraduate topics incomputer science. Springer

Nilsson M, Chisholm E, Griggs D et al. (2018) Mapping interactions between the sustainable development goals: lessons learned and ways forward. Sustain Sci 13:1489-1503

Pradhan P, Costa L, Rybski D et al. (2017) A systematic study of Sustainable Development Goal (SDG) Interactions. Earth's Future 5:1169-1179

Sachs J, Schmidt-Traub G, Kroll C et al. (2016) SDG index and dashboards-global report. Bertelsmann Stiftung and Sustainable Development Solutions Network (SDSN), New York

Sachs J, Schmidt-Traub G, Kroll C et al. (2019) Sustainable development report 2019. Bertelsmann Stiftung and Sustainable Development Solutions Network (SDSN), New York

Salvia AL, Leal Filho W, Brandli LL et al. (2019) Assessing research trends related to Sustainable Development Goals: local and global issues. J Clean Prod 208:841-849

Schmidt-Traub G, Kroll C, Teksoz K et al. (2017) National baselines for the Sustainable Development Goals assessed in the SDG Index and Dashboards. Nat Geosci 10:547-555

Scoones I, Stirling A, Abrol D et al. (2020) Transformations to sustainability: combining structural, systemic and enabling approaches. Curr Opin Environ Sustain $42: 65-75$

Shaikh MA, Hadjikakou M, Bryan BA (2021) National-level consumption-based and production-based utilisation of the land-system change planetary boundary: patterns and trends. Ecol Indic 121:106981

Stafford-Smith M, Griggs D, Gaffney O et al. (2017) Integration: the key to implementing the Sustainable Development Goals. Sustain Sci 12:911-919

Tibshirani R, Walther G, Hastie T (2001) Estimating the number of clusters in a data set via the gap statistic. J R Stat Soc B 63:411-423

United Nations (2015) Transforming our world: The 2030 agenda for sustainable development. United Nations, New York

United Nations (2017) Resolution adopted by the General Assembly on 6 July 2017. United Nations General Assembly, New York

United Nations (2021) The Sustainable Development Goals report 2021. United Nations, New York

Wackernagel M, Hanscom L, Lin D (2017) Making the sustainable development goals consistent with sustainability. Front Energy Res 5:18. https://doi.org/ 10.3389/fenrg.2017.00018

Wang Y, Yuan J, Lu Y (2020) Constructing demonstration zones to promote the implementation of Sustainable Development Goals. Geo Sustain 1:18-24

Warchold A, Pradhan P, Kropp JP (2020) Variations in sustainable development goal interactions: population, regional, and income disaggregation. Sustain Dev 29:285-299

Wei YQ, Li X, Gao F et al. (2018) The United Nations Sustainable Development Goals (SDGs) and the response strategies of China. Adv Earth Sci 33:1084-1093. (in Chinese)

Xu ZC, Chau SN, Chen XZ et al. (2020) Assessing progress towards sustainable development over space and time. Nature 577:74-78

Yillia PT (2016) Water-energy-food nexus: framing the opportunities, challenges and synergies for implementing the SDGs. Österreichische Wasser- Abfallwirtsch 68:86-98

Yoshida K (2020) CreateTableOne. Create an object summarizing both continuous and categorical variables. https://www.rdocumentation.org/packages/ tableone/versions/0.12.0/topics/CreateTableOne. Accessed 27 Jul 2021 
Zhang R, Zhou X, Rao J et al. (2017) Status and outlook of distributed clean energy Development in China. J Shenyang Inst Eng 13:289-295. 320. (in Chinese)

\section{Acknowledgements}

We are grateful for the support provided by the National Natural Science Foundation of China (No. 42041007) and the National Key Research and Development Program of China (No. 2017YFA0604701).

\section{Author contributions}

BF designed the study. JZ collected the data. JZ, SW, WZ performed most of the data analysis. BF coordinated and supervised the study. JZ and MEM drafted the manuscript. All authors reviewed the manuscript and approved it for submission.

\section{Competing interests}

The authors declare no competing interests.

\section{Ethical approval}

This article does not contain any studies with human participants performed by any of the authors.

\section{Informed consent}

This article does not contain any studies with human participants performed by any of the authors.

\section{Additional information}

Supplementary information The online version contains supplementary material available at https://doi.org/10.1057/s41599-022-01036-4.

Correspondence and requests for materials should be addressed to Bojie Fu.

Reprints and permission information is available at http://www.nature.com/reprints

Publisher's note Springer Nature remains neutral with regard to jurisdictional claims in published maps and institutional affiliations.

\begin{abstract}
cc (i) Open Access This article is licensed under a Creative Commons Attribution 4.0 International License, which permits use, sharing, adaptation, distribution and reproduction in any medium or format, as long as you give appropriate credit to the original author(s) and the source, provide a link to the Creative Commons license, and indicate if changes were made. The images or other third party material in this article are included in the article's Creative Commons license, unless indicated otherwise in a credit line to the material. If material is not included in the article's Creative Commons license and your intended use is not permitted by statutory regulation or exceeds the permitted use, you will need to obtain permission directly from the copyright holder. To view a copy of this license, visit http://creativecommons.org/ licenses/by/4.0/.
\end{abstract}

(C) The Author(s) 2022 